\title{
The Effect of Periocular Negative Pressure Application on Intraocular and Retrobulbar Pressure in Human Cadaver Eyes
}

\author{
Brian Shafer · Tanner J. Ferguson (1) · Nathan Chu $\cdot$ Enrico Brambilla \\ Paul Yoo
}

Received: October 29, 2021 / Accepted: November 29, 2021 / Published online: December 15, 2021

(c) The Author(s) 2021

\section{ABSTRACT}

Introduction: To investigate the effect of applying negative pressure (vacuum) to the periocular space on intraocular pressure (IOP) and retrobulbar pressure (RBP) by use of the Multi-Pressure Dial (MPD) system (Equinox Ophthalmic, Inc.).

Methods: Two eyes of two full body cadavers were studied. In each subject, the retrobulbar space, posterior segment and intra-goggle space were cannulated to provide direct IOP, RBP and intra-goggle pressure measurements via a pressure transducer data acquisition system. The goggles of the MPD system were placed over the eyes of each subject, and multiple test runs were performed, with negative pressure settings programmed to 5 , 10 and $20 \mathrm{mmHg}$. IOP and RBP measurements were continuously obtained during each run and plotted against time for analysis.

Supplementary Information The online version contains supplementary material available at https:// doi.org/10.1007/s40123-021-00442-2.

B. Shafer

Chester County Eye Care, Malvern, PA, USA

T. J. Ferguson ( $\square$ )

Cole Eye Institute, Cleveland, OH, USA

e-mail: tannerferg@gmail.com

N. Chu $\cdot$ E. Brambilla $\cdot$ P. Yoo

Equinox Ophthalmic, Inc., Newport Beach, CA, USA
Results: For both subjects, the mean reduction $( \pm$ standard deviation) in IOP was $1.6 \pm 0.9(10 \%)$, $3.5 \pm 1.8(23 \%)$ and $5.6 \pm 2.0(37 \%) \mathrm{mmHg}$ at programmed negative pressure levels of $-5,-10$ and $-20 \mathrm{mmHg}$, respectively. The overall mean change in RBP ( $\mathrm{mmHg}$ ) during negative pressure application was $0.02 \pm 0.14$ at $-5 \mathrm{mmHg}$, $0.03 \pm 0.19$ at $-10 \mathrm{mmHg}$ and $-0.01 \pm 0.18$ at $20 \mathrm{mmHg}$. In both subjects, the magnitude of RBP change during application of negative pressure fell below the uncertainty of the measurement system. Conclusions: The application of negative pressure to the periocular space with the MPD decreases IOP but does not affect RBP.

Keywords: CSF pressure; MPD; Multi-Pressure Dial; Normal-tension glaucoma; Open-angle glaucoma; Orbital pressure; Periocular negative pressure; Retrobulbar pressure

\section{Key Summary Points}

Why carry out this study?

The Multi-Pressure Dial (MPD) system is a removable device that delivers regulated negative pressure (vacuum) over the orbit to provide a reduction in intraocular pressure (IOP).

The use of localized, periocular negative pressure to lower IOP represents a novel approach for IOP reduction. 


\section{What was learned from this study?}

This cadaver eye study provides direct measurements confirming that regulated delivery of negative pressure to the periocular space with the MPD achieves a reduction in IOP.

This study demonstrates that application of negative pressure with the MPD does not lead to changes in retrobulbar pressure.

Negative pressure application with the MPD provides a reduction in IOP without alteration of retrobulbar pressure (RBP), allowing for a more favorable pressure differential across the posterior structures of the eye.

\section{DIGITAL FEATURES}

This article is published with digital features, including a video, to facilitate understanding of the article. To view digital features for this article, go to https://doi.org/10.6084/m9. figshare.17092118.

\section{INTRODUCTION}

Glaucoma is the world's leading cause of irreversible blindness and continues to pose a major challenge for clinicians and patients alike $[1,2]$. Current approaches to treatment target the reduction of intraocular pressure (IOP), the only clinically-validated, modifiable risk factor associated with the disease [3]. Recently, a novel IOP-lowering device known as the Multi-Pressure Dial system or MPD (Equinox Ophthalmic, Inc., Newport Beach, CA, USA) was introduced onto the market [4-6]. The MPD represents the first non-invasive, non-pharmaceutical, nonsurgical approach to IOP reduction [4, 7].

The MPD system is a removable device that employs negative pressure (vacuum) over the orbit to lower IOP. The MPD system includes a programmable pressure-modulating pump connected mechanically and pneumatically through a tubing system to specialized, pressure-sensing goggles. With activation of negative pressure, the MPD reduces the local air pressure within the goggle chambers, leading to a lowering of pressure inside the eye relative to atmospheric pressure (IOP) [8]. The operating principle of the device is based on Pascal's law, and the decreased air pressure applied to the eye generates a decrease in pressure inside the eye relative to the atmosphere surrounding the body.

The use of localized, periocular negative pressure to lower IOP represents a novel approach to reducing IOP. A prior study by Ethier et al. [8] provided insight into the mechanism of IOP reduction achievable with the MPD and created a mathematical model to investigate the impact of periocular negative pressure. The model demonstrated that the IOP quickly achieves a steady state and that the IOP reduction is maintained while negative pressure is active. Once negative pressure is deactivated, IOP quickly returns to the baseline level prior to the application of negative pressure.

Given the mechanism of IOP reduction conferred by the MPD system, the aim of the present study was to further explore the impact of localized, periocular negative pressure on intraocular, retrobulbar and intra-goggle pressures. To collect data, both intraocular and retrobulbar pressures were directly measured on two full body cadaver subjects studied in situ at variable negative pressure settings with the MPD.

\section{METHODS}

Both subjects were deceased individuals who had expired $<5 \mathrm{~h}$ prior to the beginning of the study. Ethics committee approval was not required as Institutional Review Board approval is not a requisite for cadaver studies. Appropriate consent was obtained for research purposes..

To obtain direct measurements from the intraocular, intra-goggle and retrobulbar space, the retrobulbar space, posterior segment and intra-goggle space were cannulated. From each space, individual lines were connected with three separate, disposable pressure transducers, 


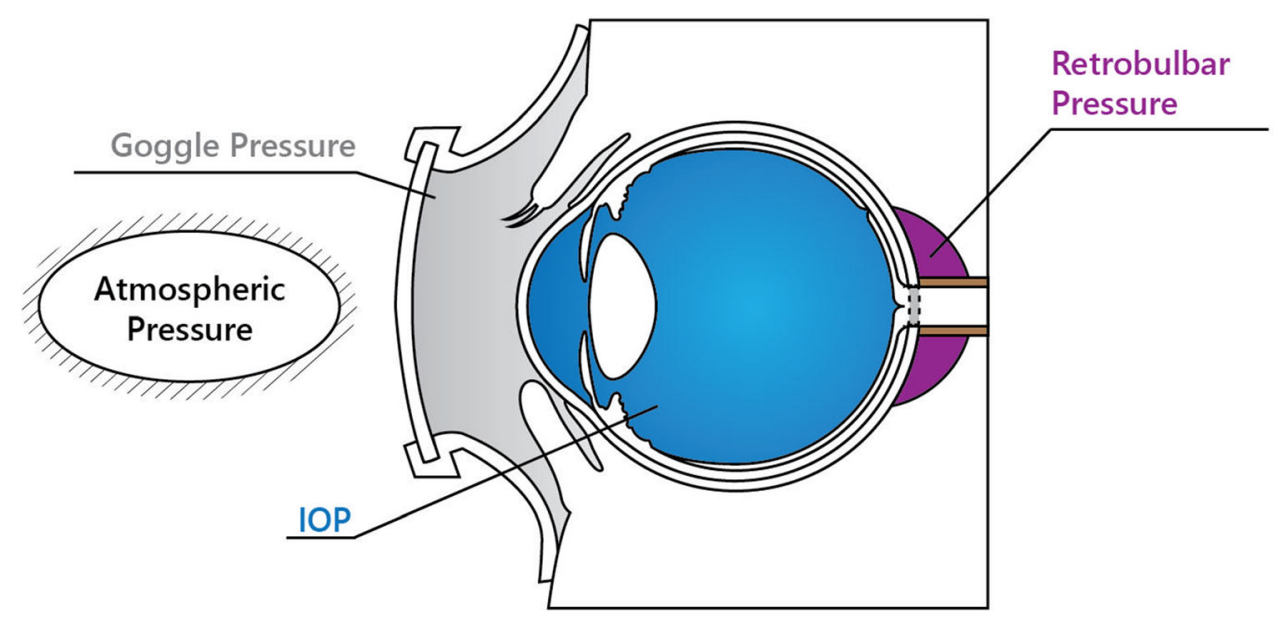

Fig. 1 Diagrammatic representation of the three pressures directly measured in this study: intraocular pressure (IOP), goggle pressure and retrobulbar pressure

and each line was aligned at eye level to maximize precision and accuracy of measurements. The three pressures (intraocular, goggle and retrobulbar) of interest in this study are shown diagrammatically in Fig. 1.

IOP values were directly measured by cannulating the posterior segment (e.g. vitreous) with a 16-gauge needle on the distal end of a fluid-filled line via a transconjunctival approach $4 \mathrm{~mm}$ posterior to the limbus. To obtain direct retrobulbar pressure (RBP) measurements, the retrobulbar space was cannulated with an 18-gauge needle on the distal end of a fluid-filled line via a transcutaneous approach. An additional line was air-filled and connected to the goggle to directly measure pressure within the MPD goggles. The positioning and setup of the ports for pressure measurement are shown in Fig. 2.

The measurement system employed three disposable pressure transducers to provide direct pressure measurements. To permit digital data acquisition from the sensors, the transducers were connected to a data acquisition (DAQ) device that was connected to a computer to allow for real-time monitoring of the IOP, RBP and intra-goggle pressure.

Prior to the application of negative pressure, the MPD goggles were fastened to the skin and anterior orbital rim using Dermatac ${ }^{\circ}$ tissue sealant (3M Company, Saint Paul, MN, USA) to ensure an airtight seal. After a proper seal was confirmed, test runs were performed with continuous pressure measurements from each of the three transducers. The data were recorded on a computer connected to the tranducers, and the individual test runs were separated for analysis. The aim of each test run was to apply negative pressure three times at each of three negative pressure levels $(-5,-10$ and -20 $\mathrm{mmHg}$ ) with the MPD.

IOP and RBP pressure tracings relative to application of negative pressure in the goggles were available in real time, allowing simultaneous comparison of RBP, IOP and intra-goggle pressure. The data from the sensors were plotted against time and presented for each run. Since pressure measurements were continuously obtained with the DAQ system, a series of measurements was averaged to generate a value for comparison and evaluation. For example, across a test run, the last 20 measurement samples prior to each event (e.g. the 20 sample measurements prior to the application of negative pressure or the 20 sample measurements prior to the release of negative pressure) were averaged to assess the magnitude of response for IOP and RBP.

Subject 1 underwent a total of four test runs. During the first test run, a gradual diminution in IOP and RBP was noted as time progressed. Over the course of the first test run, the baseline IOP (without negative pressure) gradually decreased from 19.69 to $17.31 \mathrm{mmHg}$ over the 


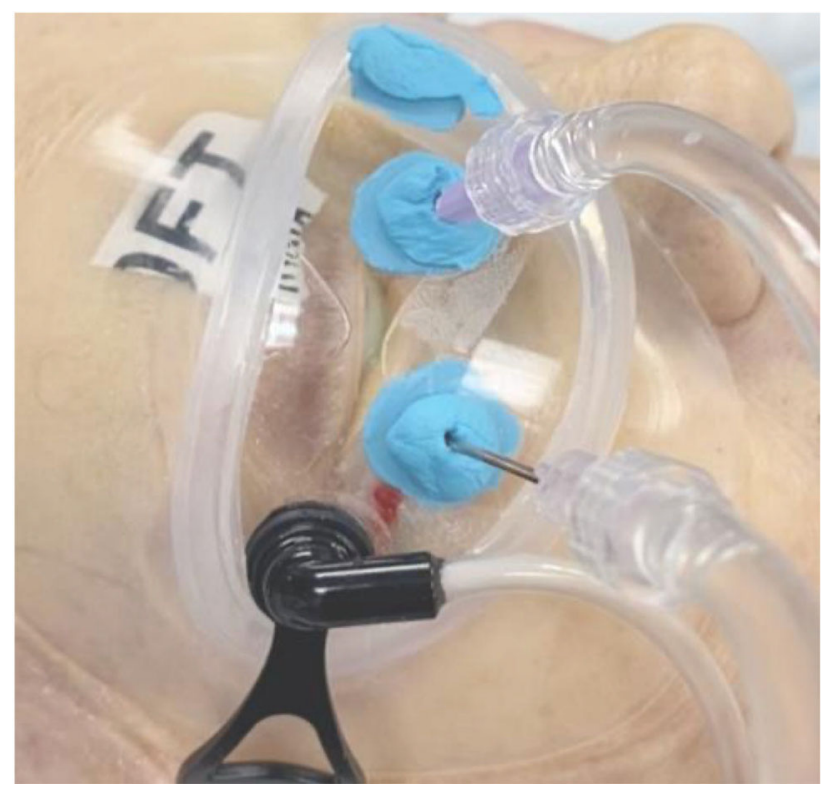

Fig. 2 The study setup. The fluid lines for both the intraocular and retrobulbar space were positioned and secured with putty to maintain a negative pressure environment within the goggles

course of the test run. In addition, the baseline RBP (without negative pressure) measurement was $1.27 \mathrm{mmHg}$ and gradually decreased to $0.42 \mathrm{mmHg}$. To attempt to compensate for the pressure decay, the IOP and RBP were increased prior to test run 2 to re-establish a baseline setting by injecting a balanced salt solution into the intraocular and retrobulbar space. Despite this effort to re-pressurize the intraocular and retrobulbar space, the small drift in IOP and RBP was still observed in between applications of negative pressure across the second and third test runs. In subject 1 , the IOP eventually stabilized at a baseline IOP of approximately $13 \mathrm{mmHg}$ prior to the fourth test run.

Subject 2 also underwent a total of four test runs. Similar to subject 1 , there was a gradual drift in IOP during the test runs in between applications of negative pressure, but no artificial pressurization was applied prior to any test runs. The baseline IOP was $15 \mathrm{mmHg}$ prior to the first test run and $13 \mathrm{mmHg}$ prior to the second test run, and then stabilized around $10 \mathrm{mmHg}$ for the third and fourth test runs. Following the second test run, an additional 1-h test run was performed to evaluate the behavior and response of the IOP and RBP when negative pressure was applied for an extended duration.
The fourth test run was thus performed following the 1-hour-long application of negative pressure.

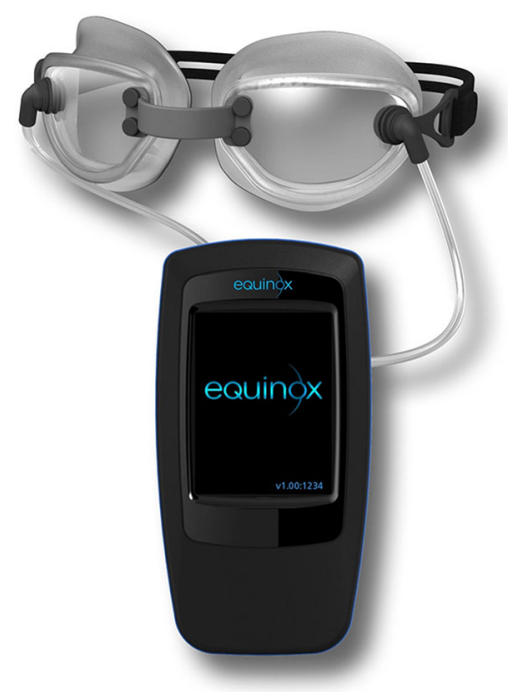

Fig. 3 The Multi-Pressure Dial (MPD) system, which consists of specialized goggles connected to a pressuremodulated pump 


\section{Multi-Pressure Dial}

The MPD is made up of two components: a pair of pressure-sensing goggles that is connected by a specialized tubing system on each side to a programmable pump. The current version of the MPD is shown in Fig. 3. The MPD goggles include an adjustable head strap for support and are designed with an anthropometric fit that enables extended wear length with negative pressure application. The programmable pump is connected to the goggles by crush-resistant tubing with separate lumens that enable independent negative pressure settings for each eye. The tubing system has separate vacuum- and pressure-sensing lines to allow a closed-loop control of the vacuum. This design permits realtime titratability of the negative pressure in addition to negative pressure sensors directly in the goggle chambers to ensure the programmed negative pressure setting is achieved.

The MPD goggles used in this present study were modified to create sealed ports used for cannulation and direct measurement of the intra-goggle, intraocular and retrobulbar space.

\section{Pressure Measurement System}

The method used for retrobulbar pressure measurement is similar to the one described in a prior study measuring orbital pressure in which a compartment pressure transducer (Compass; Mirador Biomedical Inc., Seattle, WA, USA) was utilized [9]. The pressure transducer used in the present study was also similar, but was connected to a fluid-filled line, as opposed to a fluid-filled syringe. This modification enabled continuous pressure monitoring and subsequent logging of data.

The system included multiple components. Three disposable pressure transducers (Deltran ${ }^{\circledR}$ II series, model 6199; Utah Medical Products Inc., Midvale, UT, USA) were separately connected to each space to obtain pressure measurements. Voltage was supplied to the sensors using a voltage reference component (LJTickVRef-41 [LJTVR-41] module; LabJack Corporation, Lakewood, CO, USA), and the voltage was amplified using a signal-conditioning module
(LJTick-AMP; LabJack Corporation). The entire system was connected to a USB DAQ board (model U3-HV; LabJack Corporation). The DAQ board enabled continuous sampling of the data, and the data was recorded via a connected computer. This system enabled the assessment of time-based responses relative to negative pressure application in real time.

Prior to initiation of the study, the linearity and sensitivity of the system was evaluated to ensure the pressure measurements were accurate and representative. The linearity was tested by evaluating the sensor output against a calibrated differential pressure manometer (model HD755; Extech Instruments, Nashuah, $\mathrm{NH}$, USA). The sensitivity of the system was assessed using a water column at three different pressure ranges. In addition, a "pinch test" was performed after each needle was positioned at the target location by compressing the fluid tubing and confirming a spike in pressure reading, as confirmation that the system was actively measuring pressure. The "pinch test" was repeated during the second test run of subject 1 to verify the system was patent and correctly obtaining pressure measurements, as shown in Fig. 4.

\section{RESULTS}

There were two eyes from two cadavers included in the study. Subject 1 was a 62 -year-old female, and subject 2 was a 76-year-old male. Both subjects were pseudophakic.

\section{Intraocular Pressure}

Subject 1 underwent a total of four test runs, which are shown in Fig. 5. Across all test runs, at a negative pressure setting of $-5 \mathrm{mmHg}$ with the MPD, the mean ( \pm standard deviation) IOP reduction was $2.5 \pm 0.3 \mathrm{mmHg}$ from the baseline value of $16.1 \pm 2.8 \mathrm{mmHg}$, representing a mean IOP reduction of $16 \%$ with respect to the pre-vacuum IOP. At the $-10 \mathrm{mmHg}$ negative pressure level, the mean IOP reduction was $4.8 \pm 1.3 \mathrm{mmHg}$ from a baseline of $16.9 \pm 2.4 \mathrm{mmHg}$, representing a mean reduction in IOP across all test runs of $28 \%$. At 


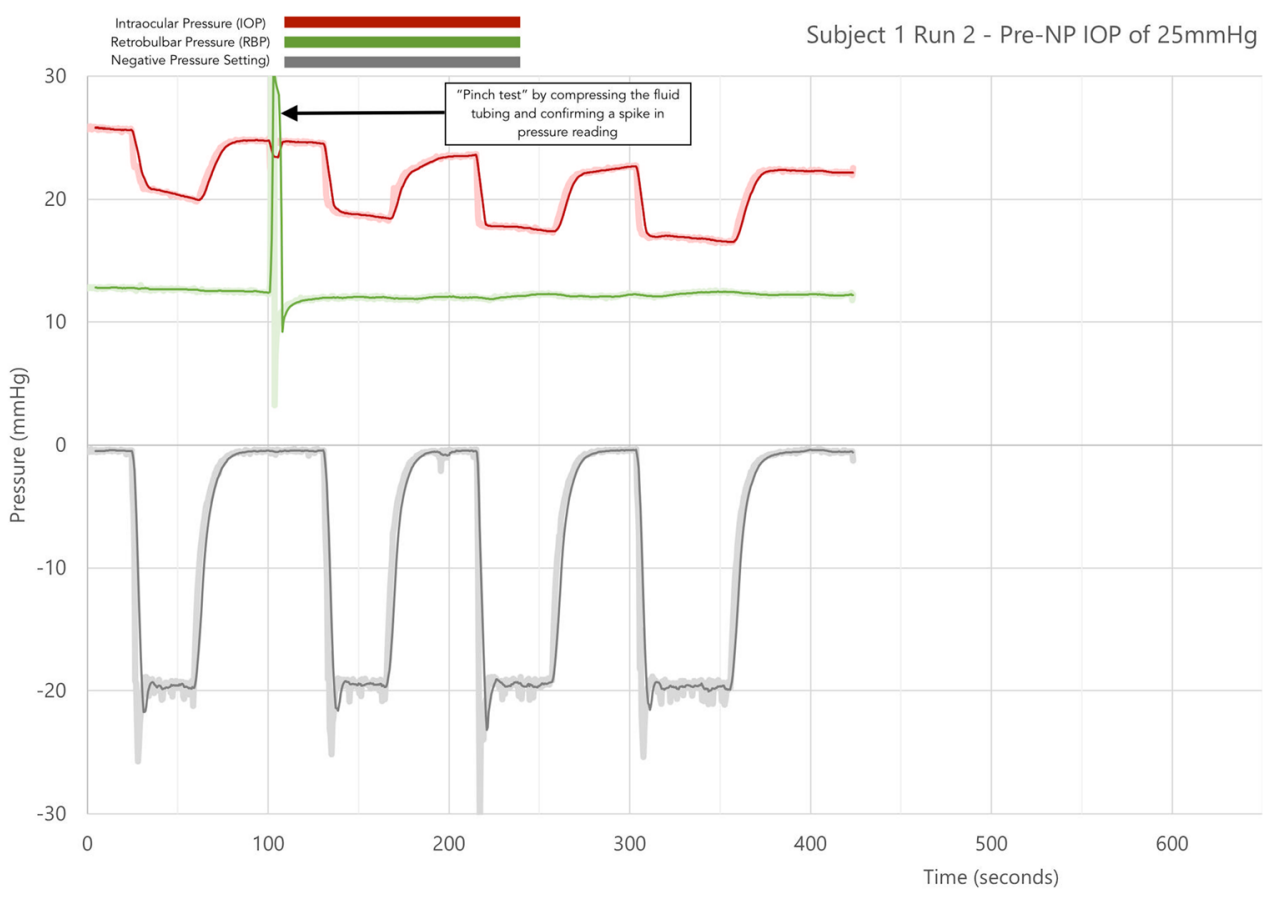

Fig. 4 The second test run of subject 1 , shown to highlight the "pinch test", which occurred at the 100-s time point in the tracing and is illustrated by the spike in

$-20 \mathrm{mmHg}$ of negative pressure, the mean reduction in IOP was $5.3 \pm 1.0 \mathrm{mmHg}$ from a baseline of $18.5 \pm 4.1 \mathrm{mmHg}$, representing a $30 \%$ mean reduction in IOP.

Subject 2 also underwent a total of four test runs, including a 1-h test run programmed at $20 \mathrm{mmHg}$. The three test runs at variable negative pressure settings are shown in Fig. 6. At a negative pressure setting of $-5 \mathrm{mmHg}$, the mean IOP reduction was $1.0 \pm 0.5 \mathrm{mmHg}$ from a baseline value of $12.9 \pm 2.4 \mathrm{mmHg}$, which represented a $7 \%$ mean reduction in IOP. At a programmed setting of $-10 \mathrm{mmHg}$, the mean reduction in IOP was $2.3 \pm 1.2 \mathrm{mmHg}$ from a baseline value of $12.5 \pm 2.2 \mathrm{mmHg}$, representing a mean reduction of $17 \%$. At test runs programmed to $-20 \mathrm{mmHg}$ of negative pressure, the mean reduction in IOP was $6.1 \pm 3.4 \mathrm{mmHg}$, from a baseline value of $11.5 \pm 1.9 \mathrm{mmHg}$, representing a mean IOP reduction of $50 \%$. The IOP results for both subjects are summarized in Table 1.

The 1-h test run was programmed to $-20 \mathrm{mmHg}$ negative pressure for the entire pressure (RBP) coinciding with the compression of fluid tubing. $N P$ Negative pressure

duration of the test run and was performed as an exploratory measure to assess the impact of sustained negative pressure on the IOP and RBP. Across the entire test run, the mean IOP reduction was $4.8 \pm 0.2 \mathrm{mmHg}$, from a baseline of $10.5 \pm 0.5 \mathrm{mmHg}$, representing a $46 \%$ reduction in IOP. The tracing of the 1-h test run is shown in Fig. 7; the magnitude of IOP reduction is clearly maintained and consistent throughout the 1 -h test run without any detectable change in RBP.

Across all test runs for both subjects, each IOP measurement with the application of negative pressure demonstrated a reduction in IOP. Overall, for all test runs in each subject, the mean baseline IOP was $17.5 \pm 3.4 \mathrm{mmHg}$ in subject 1 and $12.4 \pm 2.2 \mathrm{mmHg}$ in subject 2 . For both subjects, the overall mean reduction in IOP was $1.6 \pm 0.9 \mathrm{mmHg}$ at the $-5 \mathrm{mmHg}$ negative pressure setting, $3.5 \pm 1.8 \mathrm{mmHg}$ at the $-10 \mathrm{mmHg}$ negative pressure setting and $5.6 \pm 1.9 \mathrm{mmHg}$ at the $-20 \mathrm{mmHg}$ negative pressure setting. The mean IOP reduction for both subjects was 10,23 and $37 \%$ at the 


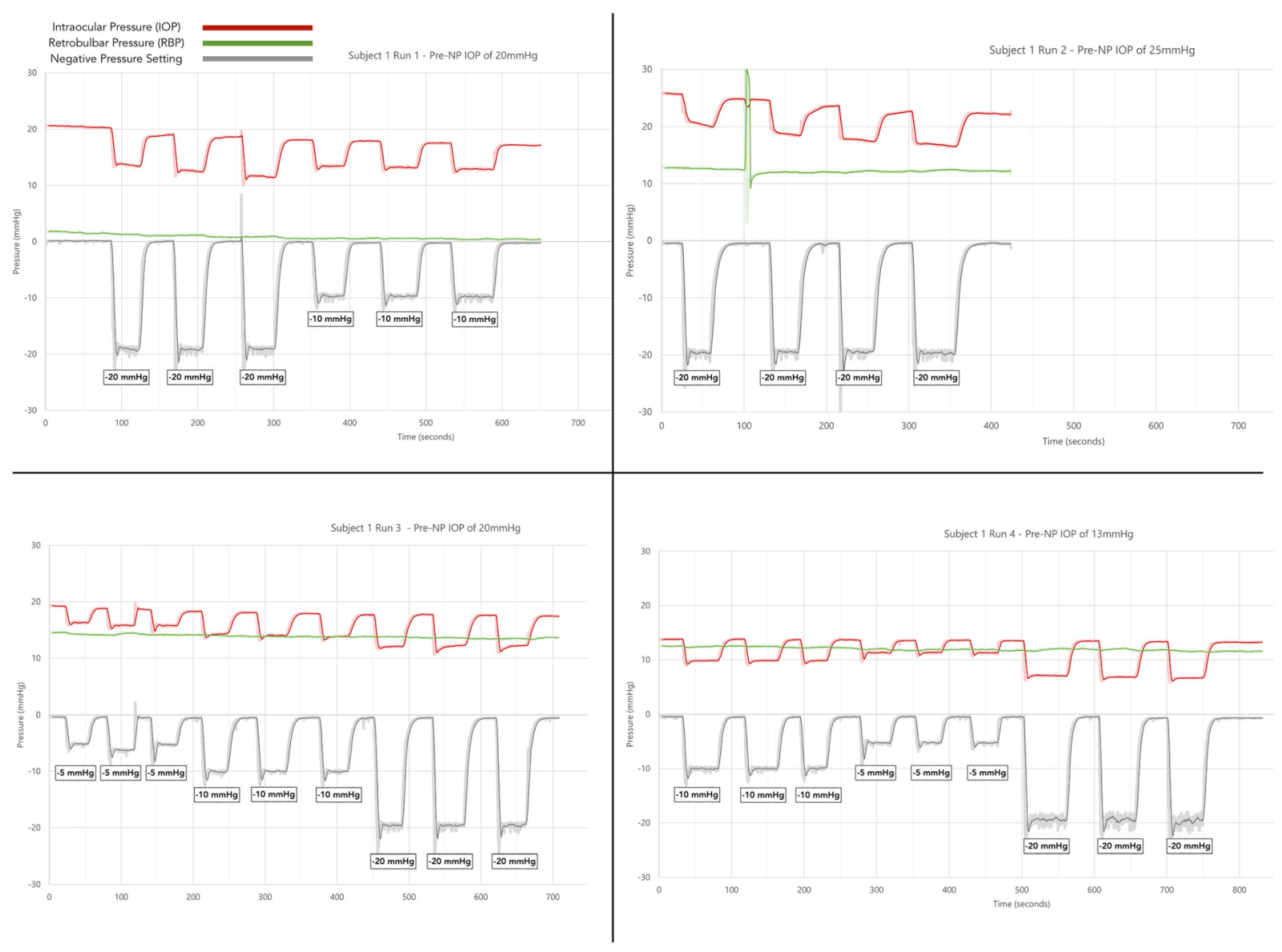

Fig. 5 Tracings of the four test runs of subject 1. The negative pressure settings are labeled in each test run to highlight the corresponding change in IOP with the application of negative pressure

programmed negative pressure levels of -5 , -10 and $-20 \mathrm{mmHg}$, respectively. For both subjects, an increase in the level of negative pressure in the goggles corresponded with an increase in the magnitude of IOP reduction at all negative pressure settings.

\section{Retrobulbar Pressure}

In subject 1 , across all test runs the overall mean change in RBP with the application of negative pressure was $+0.08 \pm 0.12 \mathrm{mmHg}$. With the application of $-5,-10$ and $-20 \mathrm{mmHg}$ of negative pressure, the mean change in RBP was $+0.06 \pm 0.14,+0.09 \pm 0.12$ and $+0.07 \pm 0.12 \mathrm{mmHg}$, respectively. For all test runs, the total magnitude of RPB change was below the uncertainty (estimated $\pm 1 \mathrm{mmHg}$ ) of the measurement system used to obtain pressure measurements.

Similar results were observed in subject 2 . For all test runs, the overall mean change in RBP with the application of negative pressure was $-0.05 \pm 0.19 \mathrm{mmHg}$. The mean change in RBP was $-0.01 \pm 0.13$ at the $5 \mathrm{mmHg}$ negative pressure setting, $-0.03 \pm 0.24 \mathrm{mmHg}$ at the - $10 \mathrm{mmHg}$ negative pressure setting and $-0.16 \pm 0.18 \mathrm{mmHg}$ at the $-20 \mathrm{mmHg}$ negative pressure setting, as regulated with the MPD. The 1-h test run was also performed in subject 2 to explore the impact of negative pressure on IOP and RBP over an extended period of time. Across the 1-h duration of the test run, there was no visual change apparent in RBP, and the 

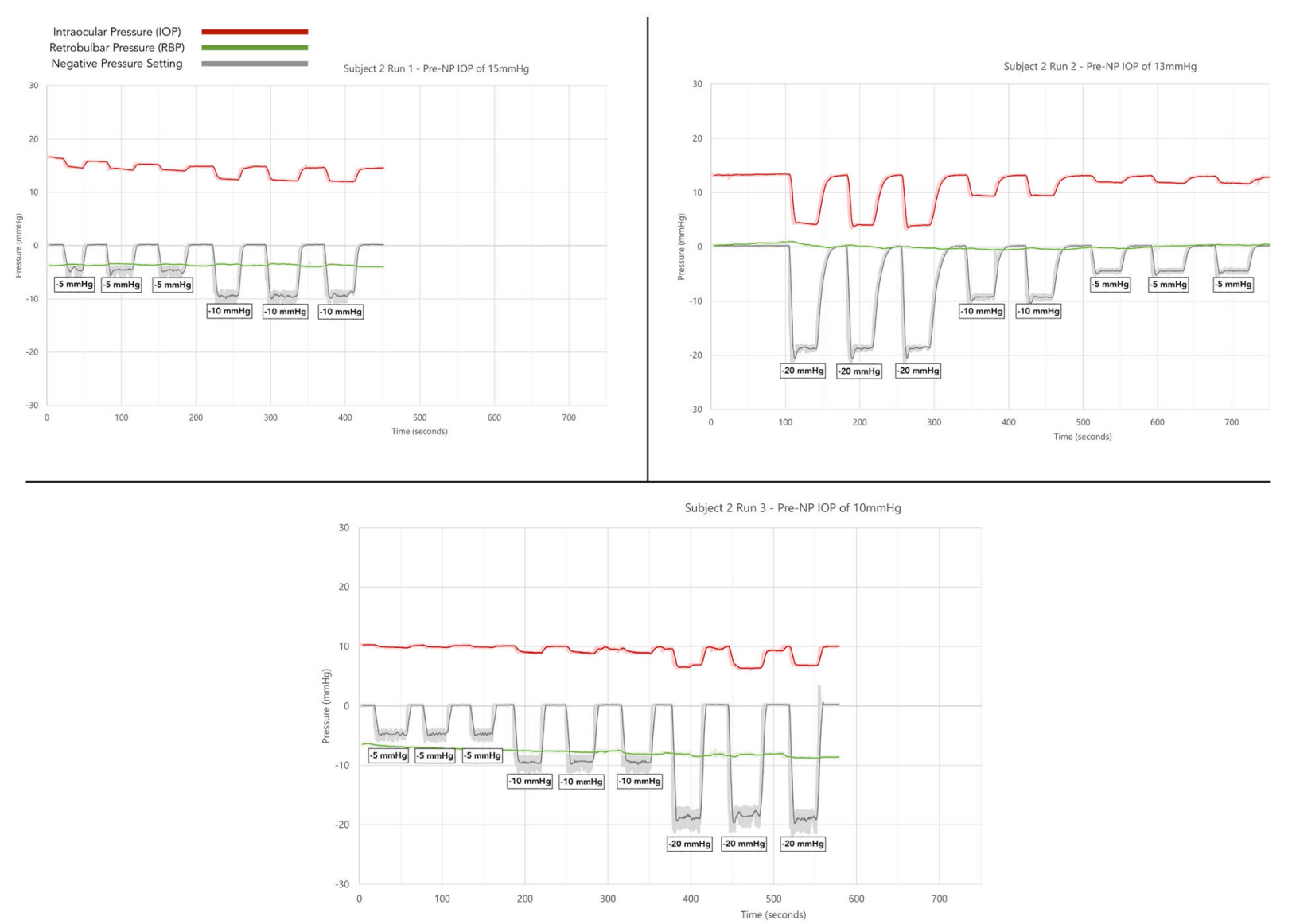

Fig. 6 The three test runs of subject 2 conducted at variable negative pressure settings $(-5,-10$, $20 \mathrm{mmHg}$ ). The 1-h test run is not included (see Fig. 7).

mean change in RBP was $0.13 \pm 0.54 \mathrm{mmHg}$. The RBP results are summarized in Table 2.

For both subjects, the overall mean change in RBP fell below the uncertainty of the measurement, indicating that there was no detectable change in RBP beyond the accuracy of the measurement system.

\section{DISCUSSION}

The aim of this study was to evaluate the impact of periocular negative pressure on intraocular and retrobulbar pressures using the MPD, a novel device that employs regulated, periocular negative pressure to lower IOP. In both subjects, pressure measurements obtained via direct cannulation to the retrobulbar space revealed
The negative pressure settings are labeled in each test run to highlight the corresponding change in IOP with the application of negative pressure

no significant change in the RBP pressure as negative pressure was applied and released, supporting the safety of the MPD.

This study also demonstrates that negative pressure application to the periocular space consistently lowers IOP as measured via direct pressure transducer pressure measurements. In both subjects, there was a reduction in IOP with application of negative pressure that promptly returned to baseline with cessation of negative pressure. Further, there was no evidence of rebound IOP spikes following the application of negative pressure across all test runs. The results of this study confirm that controlled application of periocular negative pressure leads to a reduction in IOP, supporting the results of prior clinical studies $[6,7,10]$ that reported a reduction in IOP with the MPD system. Collectively, 
Table 1 Baseline intraocular pressure (IOP), IOP following application of negative pressure and mean change in IOP following application of negative pressure for each subject

\begin{tabular}{|c|c|c|c|c|}
\hline $\begin{array}{l}\text { Negative } \\
\text { pressure setting }\end{array}$ & $\begin{array}{l}\text { Baseline IOP }(\mathrm{mmHg}) \\
(\text { mean } \pm \text { SD })\end{array}$ & $\begin{array}{l}\text { IOP }(\mathrm{mmHg}) \text { with negative } \\
\text { pressure }(\text { mean } \pm \mathrm{SD})\end{array}$ & $\begin{array}{l}\Delta \text { IOP }(\mathrm{mmHg}) \\
(\text { mean } \pm \mathrm{SD})\end{array}$ & $\begin{array}{l}\Delta \text { IOP } \\
(\text { mean \%) }\end{array}$ \\
\hline \multicolumn{5}{|l|}{ Subject 1} \\
\hline$-5 \mathrm{mmHg}$ & $16.1 \pm 2.8$ & $13.6 \pm 2.6$ & $-2.5 \pm 0.3$ & $-16 \%$ \\
\hline$-10 \mathrm{mmHg}$ & $16.9 \pm 2.4$ & $12.1 \pm 1.8$ & $-4.8 \pm 1.3$ & $-28 \%$ \\
\hline$-20 \mathrm{mmHg}$ & $18.5 \pm 4.1$ & $13.2 \pm 4.4$ & $-5.3 \pm 1.0$ & $-30 \%$ \\
\hline \multicolumn{5}{|l|}{ Subject 2} \\
\hline$-5 \mathrm{mmHg}$ & $12.9 \pm 2.4$ & $11.9 \pm 2.0$ & $-1.0 \pm 0.5$ & $-7 \%$ \\
\hline$-10 \mathrm{mmHg}$ & $12.5 \pm 2.2$ & $10.2 \pm 1.5$ & $-2.3 \pm 0.2$ & $-17 \%$ \\
\hline$-20 \mathrm{mmHg}$ & $11.5 \pm 1.9$ & $5.4 \pm 1.5$ & $-6.1 \pm 3.4$ & $-50 \%$ \\
\hline
\end{tabular}

Both the mean $\pm \mathrm{SD}$ and mean percentage change are shown for each negative pressure setting $(-5,-10,-20 \mathrm{mmHg})$ $\triangle$ Change, $S D$ standard deviation

Table 2 Baseline retrobulbar pressure (RBP), RBP with application of negative pressure and mean change in RBP with application of negative pressure for each subject

\begin{tabular}{llll}
\hline Negative pressure setting & Baseline RBP $(\mathbf{m m H g})$ & $\mathbf{R B P}(\mathbf{m m H g})$ with negative pressure & $\Delta \mathbf{R B P}(\mathbf{m m H g})$ \\
\hline Subject 1 & & & \\
$-5 \mathrm{mmHg}$ & $13.01 \pm 1.30$ & $13.08 \pm 1.31$ & $0.06 \pm 0.14$ \\
$-10 \mathrm{mmHg}$ & $9.05 \pm 6.11$ & $9.13 \pm 6.05$ & $0.09 \pm 0.12$ \\
$-20 \mathrm{mmHg}$ & $9.72 \pm 5.30$ & $9.80 \pm 5.31$ & $0.07 \pm 0.12$ \\
Subject 2 & & & \\
$-5 \mathrm{mmHg}$ & $-3.54 \pm 3.14$ & $-3.55 \pm 3.13$ & $-0.01 \pm 0.13$ \\
$-10 \mathrm{mmHg}$ & $-3.85 \pm 3.26$ & $-3.89 \pm 3.32$ & $-0.03 \pm 0.24^{\circ}$ \\
$-20 \mathrm{mmHg}$ & $-4.07 \pm 4.65$ & $-4.24 \pm 4.66$ & $-0.16 \pm 0.18$ \\
\hline
\end{tabular}

All RBP values are reported as the mean $\pm \mathrm{SD}$

The mean change $(\Delta)$ is demonstrated for each negative pressure setting in each subject

the decrease in IOP observed in the absence of pressure changes in the retro-orbital tissue indicates that the MPD system is capable of favorably modifying the translaminar pressure difference, a pressure differential shown to be important in the pathogenesis of glaucoma [11-14].

It is important to note the pressure measurement system employed in this study includes multiple components that collectively contributes uncertainties to the pressure measurement capability of the system. It was noted that the further the pressure measurement was from zero, the greater the magnitude of uncertainty. For example, at $30 \mathrm{mmHg}$, the theoretical measurement uncertainty was $\pm 0.813 \mathrm{mmHg}$. Without taking into consideration other sources of noise, the accuracy of the measurement system for this study was conservatively estimated to be $\pm 1 \mathrm{mmHg}$. 


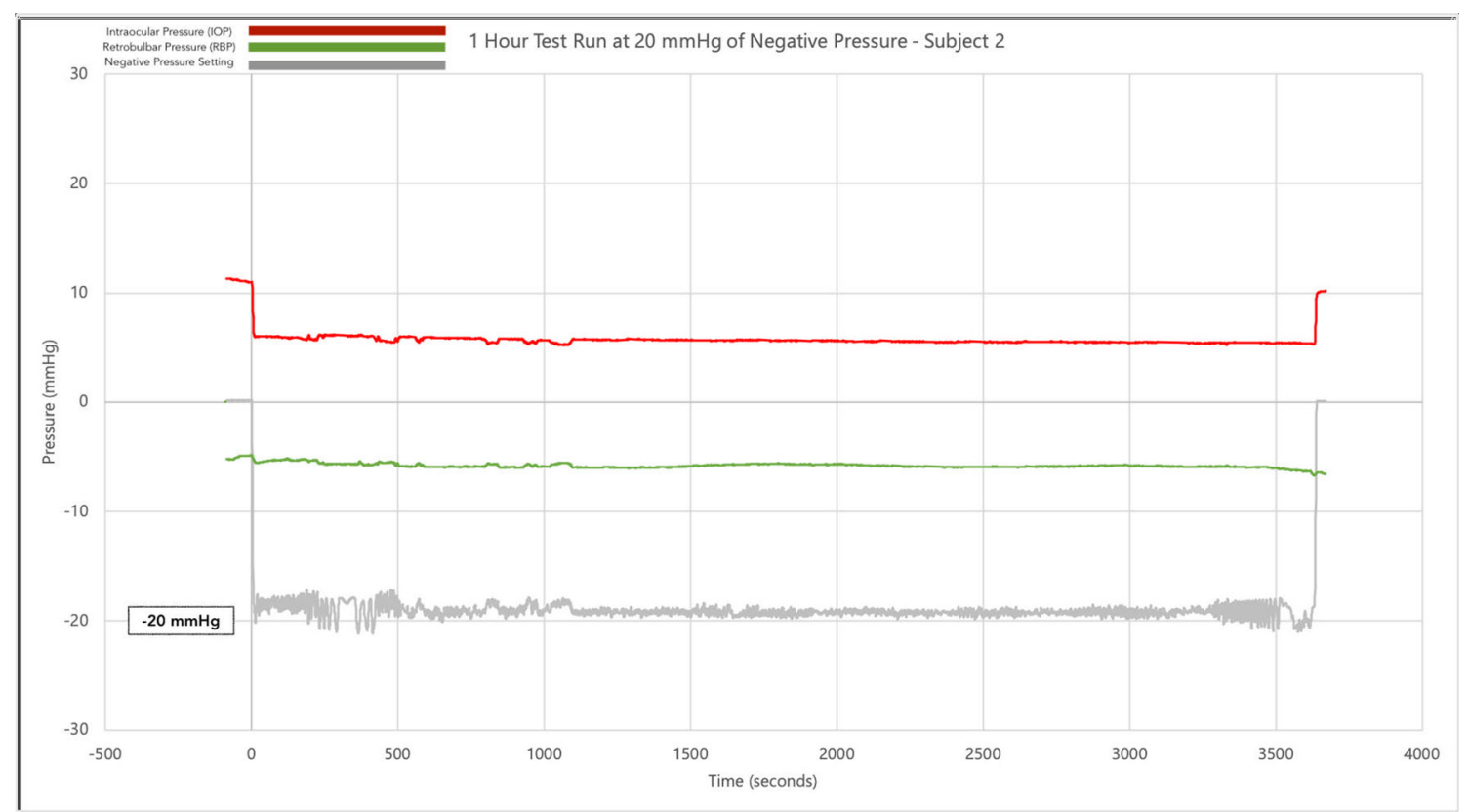

Fig. 7 The 1-h test run for subject 2. This test run was performed to evaluate the behavior of both IOP and RBP during the application of negative pressure for an extended period of time. No visual change in RBP is apparent

Given that the changes in RBP fell below the range of uncertainty in both subjects, we concluded there was no detectable change in RBP with application of negative pressure across all test runs.

Multiple prior studies have investigated the IOP-lowering ability of the MPD in both healthy and glaucomatous eyes as measured via the excursion test method, a developed technique for IOP measurement that involves a pneumatonometer across a tonometer tip cover (Tono-pen ${ }^{\circledR}$; Reichert Technologies, Buffalo, New York) with a specially adapted version of the MPD goggles $[6,7,10]$. In a recent study in 65 healthy subjects, Swan et al. [6] used three different negative pressure settings, namely 25 , 50 and $75 \%$ of baseline IOP, and demonstrated a stepwise increase in IOP reduction with increased levels of negative pressure. In this study, at the $75 \%$ negative pressure setting, the mean IOP was reduced to $10.2 \pm 2.7 \mathrm{mmHg}$, from a baseline of $15.8 \pm 3.6 \mathrm{mmHg}$, which is a $35 \%$ reduction in IOP. The results of this study support the capability of the MPD system to provide a meaningful reduction in IOP even in patients with lower baseline IOP. An additional, recently published study by Goldberg et al. [7] assessed the nocturnal IOP-lowering ability of the device by assessing IOP measurements at three separate time points throughout the night in subjects with open-angle glaucoma who were in the supine position. At all three time points (10:30 p.m., 2:00 a.m., 5:30 a.m.), the IOP reduction exceeded 30\% while the MPD was worn with a negative pressure setting programmed to $60 \%$ of baseline IOP for all subjects. Further, the IOP reduction demonstrated in this study occurred in subjects actively on a topical prostaglandin medication, indicating that the MPD can provide an additive IOP-lowering effect.

The IOP-lowering results demonstrated in this present study align with the results reported in prior clinical studies that have evaluated the IOP-lowering ability of the MPD $[6,7,10]$. In both subjects in the present study, an increase in the programmed level of negative pressure within the goggles corresponded to an increased reduction in IOP. For example, at $-5 \mathrm{mmHg}$ of programmed negative pressure, 
the overall mean IOP reduction for both subjects was $1.57 \pm 0.87 \mathrm{mmHg}$; at $-20 \mathrm{mmHg}$ of negative pressure, which is the highest level of negative pressure employed in this study, the mean IOP reduction was $5.58 \pm 1.98 \mathrm{mmHg}$, more than triple the magnitude of response observed at $-5 \mathrm{mmHg}$. Moreover, the mean IOP reduction at $-20 \mathrm{mmHg}$ of negative pressure was $37 \%$, a meaningful degree of IOP reduction given the lower baseline IOP. In addition, the live tracings (Figs. 5-7) highlight the transient and instantaneous action of the MPD by demonstrating the reversibility of IOP back to baseline with the application and release of negative pressure.

A 1-h-long test run was performed in subject 2 to assess the response of the RBP and IOP with the application of $-20 \mathrm{mmHg}$ negative pressure over an extended period of time. Over the 1-h time frame, there was a mean reduction in IOP of $4.8 \pm 0.2 \mathrm{mmHg}$. In contrast, there was no change in the RBP along the tracing, and the overall magnitude of RBP change was $0.13 \pm 0.54 \mathrm{mmHg}$, indicating there was no meaningful change in RBP relative to the system's uncertainty. These results support the safety profile of the MPD and indicate that sustained application of negative pressure provides a sustained reduction in IOP without impacting the pressure behind the eye.

This study is not without limitations. This was a small series of two cadaver subjects. In both cadavers, there was a gradual diminution of the IOP as time progressed, possibly due to absence of aqueous production or leakage. The gradual decay of baseline IOP may have affected the maximal amplitude of IOP response relative to negative pressure application, but since the IOP decrease was measured relative to the immediate baseline IOP preceding negative pressure application, this IOP drift did not otherwise have an impact on the results of this study. In addition, the IOP-lowering response observed in this study is consistent with the IOP reduction demonstrated in live human subjects $[6,10]$. Further, for both cadavers, $100 \%$ of the direct IOP measurements obtained during application of negative pressure represented a reduction in IOP.

\section{CONCLUSION}

This study corroborates the existing literature and provides direct measurements confirming that regulated delivery of negative pressure to the periocular space provides a reduction in IOP. In addition, the results of this study demonstrate that application of negative pressure does not lead to changes in RBP, and the 1-h test run without evidence of RBP change supports the safety of sustained negative pressure application. Overall, the reduction of IOP without concomitant changes in RBP allows for a more favorable pressure differential across the posterior structures of the eye.

\section{ACKNOWLEDGEMENTS}

Funding. Sponsorship for this study and the journal's Rapid Service Fees were funded by Equinox Ophthalmic Inc., Newport Beach, CA.

Authorship. All named authors meet the International Committee of Medical Journal Editors (ICMJE) criteria for authorship for this article, take responsibility for the integrity of the work as a whole, and have given their approval for this version to be published.

Author Contributions. Brian M Shafer, Tanner J Ferguson, Nathan Chu, Enrico Brambilla and Paul Yoo all equally contributed to study concept and design, writing of the original draft and review/editing of the final draft of the manuscript.

Disclosures. Brian M Shafer and Tanner J Ferguson are paid consultants for Equinox Ophthalmic, Inc. Nathan Chu, Enrico Brambilla and Paul Yoo are employees of Equinox Ophthalmic, Inc.

Compliance with Ethics Guidelines. Ethics committee approval was not required as IRB approval is not a requisite for cadaver studies. Appropriate consent was obtained for research purposes. 
Data Availability. The datasets generated during and/or analyzed during the current study are available from the corresponding author on reasonable request.

Open Access. This article is licensed under a Creative Commons Attribution-NonCommercial 4.0 International License, which permits any non-commercial use, sharing, adaptation, distribution and reproduction in any medium or format, as long as you give appropriate credit to the original author(s) and the source, provide a link to the Creative Commons licence, and indicate if changes were made. The images or other third party material in this article are included in the article's Creative Commons licence, unless indicated otherwise in a credit line to the material. If material is not included in the article's Creative Commons licence and your intended use is not permitted by statutory regulation or exceeds the permitted use, you will need to obtain permission directly from the copyright holder. To view a copy of this licence, visit http://creativecommons.org/licenses/by$\mathrm{nc} / 4.0 /$.

\section{REFERENCES}

1. Tham Y-C, Li X, Wong TY, Quigley HA, Aung T, Cheng C-Y. Global prevalence of glaucoma and projections of glaucoma burden through 2040: a systematic review and meta-analysis. Ophthalmology. 2014;121(11):2081-90. https://doi.org/10. 1016/j.ophtha.2014.05.013.

2. Kingman S. Glaucoma is second leading cause of blindness globally. Bull World Health Organ. 2004;82:887-8. https://doi.org/10.1590/S004296862004001100019 .

3. Heijl A, Leske MC, Bengtsson B, et al. Reduction of intraocular pressure and glaucoma progression: results from the Early Manifest Glaucoma Trial. Arch Ophthalmol. 2002;120(10):1268-79.

4. Thompson VM, Ferguson TJ, Ahmed IIK, et al. Short-term safety evaluation of a multi-pressure dial: a prospective, open-label, non-randomized study. Ophthalmol Ther. 2019;82(11):887-9. https://doi.org/10.1007/s40123-019-0181-y.
5. Samuelson TW, Ferguson TJ, Radcliffe NM, et al. 8 hrs safety evaluation of a multi-pressure dial in eyes with glaucoma: prospective, open-label randomized study. Clin Ophthalmol. 2019;13:1947-53. https:// doi.org/10.2147/OPTH.S217736.

6. Swan RJ, Ferguson TJ, Shah M, et al. Evaluation of the IOP-lowering effect of a multi-pressure dial at different negative pressure settings. Transl Vis Sci Technol. 2020;9(12):19. https://doi.org/10.1167/ tvst.9.12.19.

7. Goldberg JL, Jiminez-Roman J, Hernandez-Oteyza A, Quiroz-Mercado H. Short-term evaluation of negative pressure applied by the multi-pressure dial system to lower nocturnal IOP: prospective, controlled intra-subject study. Ophthalmol Ther. 2021;10:349-58.

8. Ethier CR, Yoo P, Berdahl JP. The effects of negative periocular pressure on intraocular pressure. Exp Eye Res. 2020;191:107928. https://doi.org/10.1016/j. exer.2020.107928.

9. Czyz CN, Strand AT. Minimally invasive in vivo orbital pressure measurement. Clin Exp Ophthalmol. 2016;44(8):724-5.

10. Ferguson TJ, Radcliffe NM, Van Tassel SH, et al. Overnight safety evaluation of a multi-pressure dial in eyes with glaucoma: prospective, open-label randomized study. Clin Ophthalmol. 2020;14: 2739-46. https://doi.org/10.2147/OPTH.S256891.

11. Berdahl JP, Fautsch MP, Stinnett SS, Allingham RR. Intracranial pressure in primary open angle glaucoma, normal tension glaucoma, and ocular hypertension: a case-control study. Invest Ophthalmol Vis Sci. 2008;49(12):5412-8. https://doi. org/10.1167/iovs.08-2228.

12. Berdahl JP, Allingham RR, Johnson DH. Cerebrospinal fluid pressure is decreased in primary open-angle glaucoma. Ophthalmology. 2008;115(5):763-8. https://doi.org/10.1016/j. ophtha.2008.01.013.

13. Berdahl JP, Ferguson TJ, Samuelson TW. Periodic normalization of the translaminar pressure gradient prevents glaucomatous damage. Med Hypotheses. 2020;144:110258.

14. Price DA, Harris A, Siesky B, Mathew S. The influence of translaminar pressure gradient and intracranial pressure in glaucoma: review. J Glaucoma. 2020;29(2):141-6. https://doi.org/10.1097/ IJG.0000000000001421. 